the patients were discussed at an MDT when they experienced subsequent relapses indicating reduced access to a multidisciplinary care. BRCA mutation status was considered the most important biomarker. Whilst HRD status was also considered important, at the time of the survey this was not routinely assessed, highlighting issues with test availability. The use of active surveillance was expected to decrease in favour of targeted therapies such as PARPi as the treatment pathway evolves.

\section{EFFECT OF SURGICAL PARADIGM CHANGE ON OVERALL SURVIVAL IN PATIENTS WITH ADVANCED EPITHELIAL OVARIAN CANCER}

N Norppa*, S Saarelainen, S Staff, A Auranen. Tampere University Hospital, Department of Obstetrics and Gynecology, Tampere, Finland

\subsection{6/ijgc-2021-ESG0.340}

Introduction/Background* Ultra-radical procedures have been introduced into surgical treatment of advanced epithelial ovarian cancer to better reach the goal of resection of all visible tumor from the abdominal cavity. This has enabled surgical treatment for patients with advanced and more widespread disease. The aim of this study was to evaluate the effect of this change in surgical paradigm on overall survival.

Methodology We collected retrospectively 247 patients with FIGO Stage IIIB-IVB ovarian, tubal and primary peritoneal carcinoma operated between 2013 and 2019 either by primary or interval cytoreduction in Tampere University Hospital. Patients were categorized into two groups and compared based on the date of the operation. Group 1 was operated between January 2013 and February 2016. Group 2 was operated between March 2016 and March 2019, during which time the change in surgical approach occurred. Groups were similar in age, ASA classification, tumor histology and primary vs interval debulking surgery rate. Patients were followed up to November 2020.

Result(s)* Complete cytoreduction (R0) increased from 14\% to $54 \%$ in Stage III patients and from $23 \%$ to $49 \%$ in Stage IV patients after the change in surgical approach towards ultraradical surgery. The proportion rate of Stage IV patients increased slightly from $31 \%$ in Group 1 to $40 \%$ in Group 2 $(p=0.145)$. The median follow-up was 28.7 months (0.592.7). In all stages combined, the median OS increased from 33.5 months in Group 1 to 54.5 months in Group 2 $(p=0.028)$. The median OS for stage III patients in Group 1

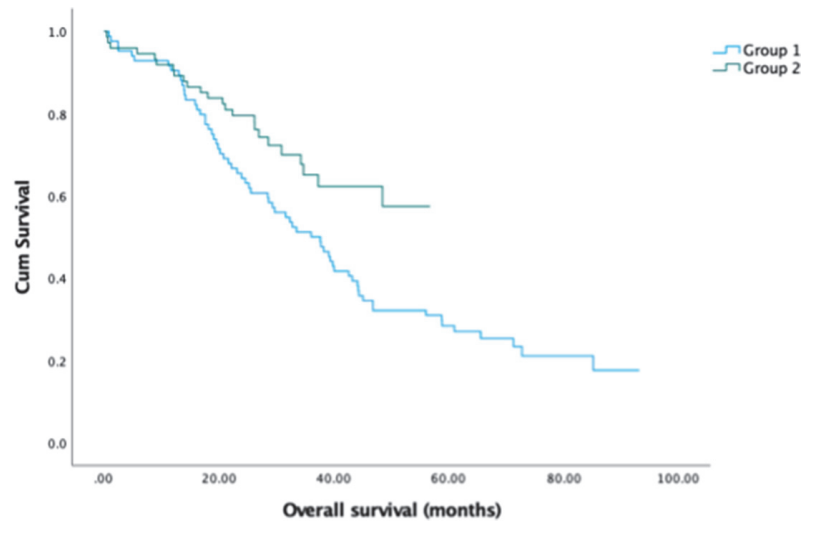

Abstract 121 Figure 1

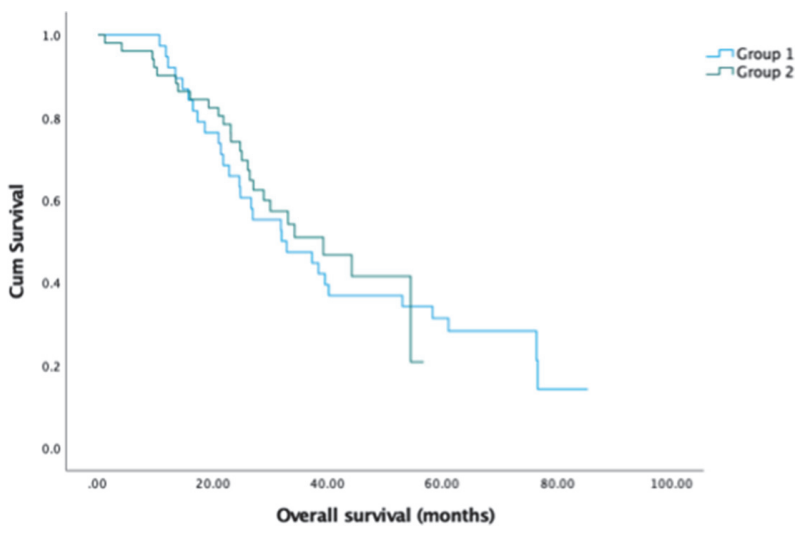

Abstract 121 Figure 2

was 36.1 months $(27.4$ - 44.8) but could not be reached in Group $2(\mathrm{p}=0.009)$. In Stage IV patients, OS was 32.0 months (16.4 - 47.7) and 39.3 months $(24.8$ - 53.8) in Group 1 and 2, respectively $(\mathrm{p}=0.691)$.

Conclusion* The change of surgical approach towards ultraradical techniques improves overall survival of patients with advanced epithelial ovarian cancer, but the survival benefit is only seen in stage III patients.

\section{PREOPERATIVE IMAGING ASSESSMENT OF PERITONEAL CANCER INDEX (PCI): CONCORDANCE WITH SURGICAL FINDINGS IN ADVANCED OVARIAN CANCER. A PROSPECTIVE STUDY}

${ }^{1} \mathrm{~S}$ Greggi*, ${ }^{2} \mathrm{O}$ Catalano, ${ }^{2} \mathrm{SV}$ Setola, ${ }^{1} \mathrm{G}$ Casella, ${ }^{1} \mathrm{G}$ Laurelli, ${ }^{1} \mathrm{~F}$ Scala, ${ }^{3} \mathrm{~V}$ Simeon, ${ }^{2} \mathrm{C}$ Granata, ${ }^{2} \mathrm{~A}$ Petrillo, ${ }^{1} \mathrm{C}$ Scaffa. ${ }^{1}$ Istituto Nazionale Tumori IRCCS "Fondazione G. Pascale", Department of Gynecologic Oncology, Naples, Italy; ${ }^{2}$ Istituto Nazionale Tumori IRCCS "Fondazione G. Pascale", Department of Radiology, Naples, Italy; ${ }^{3}$ Università degli Studi della Campania "Luigi Vanvitelli", Department of Medical Statistics, Naples, Italy

\subsection{6/ijgc-2021-ESGO.341}

Introduction/Background* The extent of peritoneal spread in advanced ovarian cancer (AOC) heavily impacts on the chance of a complete surgical cytoreduction. The decision-making process on the feasibility of cytoreductive surgery should include a dedicated imaging evaluation. The aim of this study was to prospectively compare a radiologic Peritoneal Cancer Index (rPCI) with the surgical PCI (sPCI).

Methodology 128 consecutive AOC patients planned for cytoreductive surgery underwent preoperative contrast-enhanced Computed Tomography (CT) scan to calculate the rPCI, then the sPCI was determined intraoperatively. CT scans were performed by two dedicated radiologists, and re-evaluated by a third. The rPCI correlation with sPCI was calculated by Lin's Concordance Correlation Coefficient (CCC), and represented by Bland-Altman agreement plot and Passing-Bablok regression line. Result(s)* Primary debulking surgery (PDS), and interval debulking surgery (IDS) were performed in 88 and 40 patients, respectively (complete cytoreduction in $56.8 \%$ PDS and $67.5 \%$ IDS). Overall, mean \pm SD rPCI was 16.2 \pm 6.4 (95\%CI:15.117.3) and sPCI 14.7 \pm 6.9 (95\%CI:13.5-15.9), showing a moderate correlation between preoperative CT scan and surgical findings (figures 1-2, CCC $=0.64$ ). The best concordance was reported for PDS vs. IDS (CCC $=0.64$ vs. 0.60$)$ and in middlehigh abdominal vs. low quadrants $(\mathrm{CCC}=0.57$ vs. 0.40$)$, while rPCI overestimated ileo-jejunal spread $(C C C=0.21)$. 


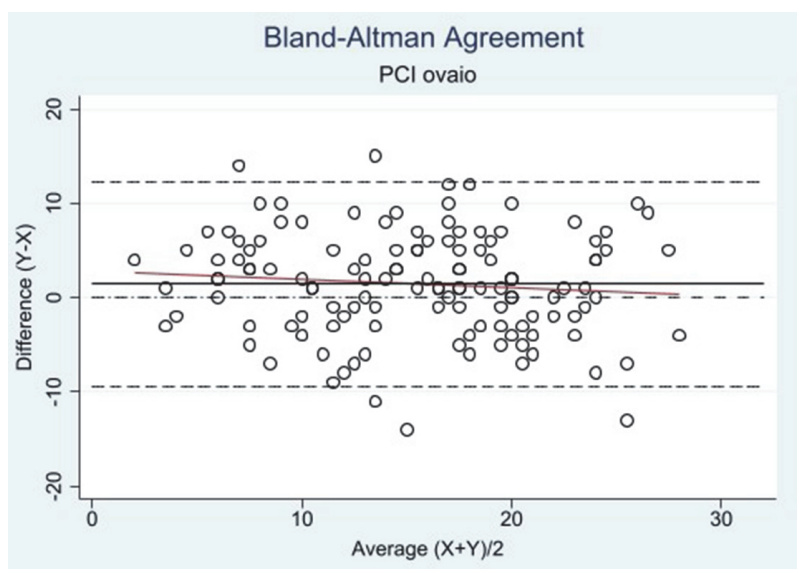

Abstract 122 Figure 1

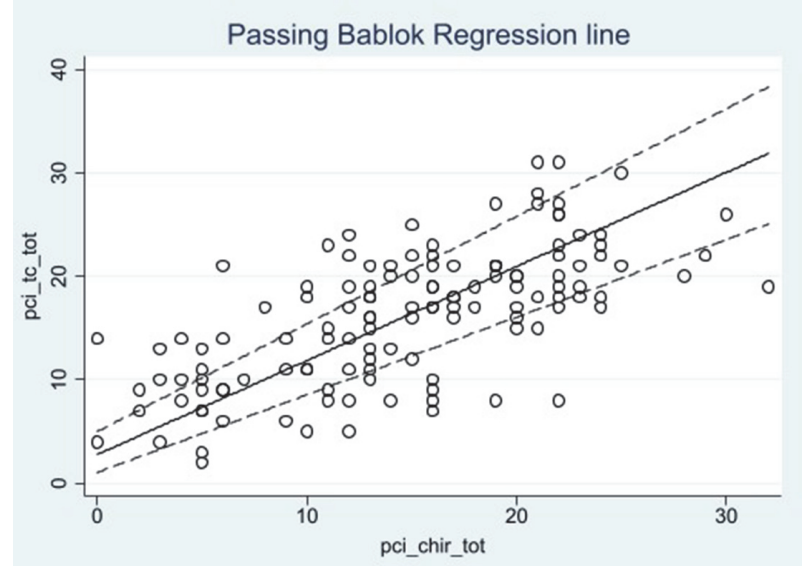

Abstract 122 Figure 2

Conclusion* CT is moderately accurate in predicting the sPCI in AOC patients. The rPCI scoring seems to be helpful and should be part of the decision-making process on surgical cytoreduction.

\section{REAL WORLD DATA OF TREATMENT AND OUTCOME OF PATIENTS WITH HIGH GRADE AOC (ADVANCED OVARIAN CANCER) IN GERMANY (QS OVAR)}

${ }^{1} \mathrm{P}$ Harter ${ }^{*},{ }^{1} \mathrm{~A}$ Du Bois, ${ }^{2} \mathrm{~F}$ Hilpert, ${ }^{3} \mathrm{M}$ Kerkmann, ${ }^{4}$ J Sehouli, ${ }^{5} \mathrm{~S}$ Mahner, ${ }^{6} \mathrm{~N}$ De Gregorio, ${ }^{7} \mathrm{~L}$ Hanker, ${ }^{1} \mathrm{~F}$ Heitz, ${ }^{8} \mathrm{~F}$ Marmé, ${ }^{2,9} \mathrm{~L}$ Wölber, ${ }^{3} \mathrm{~L}$ Holtmann, ${ }^{10} \mathrm{j}$ Pfisterer. ${ }^{1}$ Ev. Kliniken EssenMitte, Gynecology and Gynecologic Oncology, Essen, Germany; ${ }^{2}$ Jerusalem Krankenhaus, Onkologisches Therapiezentrum, Hamburg, Germany; ${ }^{3} \mathrm{MMF}$ GmbH, Dortmund, Germany; ${ }^{4}$ Charité , Klinik für Gynäkologie mit Zentrum für onkologische Chirurgie, Berlin, Germany; ${ }^{5}$ LMU University Hospital, Obstetrics and Gynecology, Munich, Germany; ${ }^{6}$ SLK Kliniken, Frauenklinik, Heilbronn, Germany; ' Universitätsklinikum Schleswig-Holstein, Campus Lübeck, , Gynecology and Obstetrics, Lübeck, Germany; ${ }^{8}$ Medical Faculty Mannheim, Heidelberg University, Frauenklinik, Mannheim, Germany; ${ }^{9}$ University Medical Center Hamburg-Eppendorf, Gynecology, Hamburg, Germany; ${ }^{10}$ Zentrum für Gynäkologische Onkologie, Kiel, Germany

\subsection{6/ijgc-2021-ESG0.342}

Introduction/Background* Recent and detailed data regarding treatment quality and outcome of patients with high grade AOC on a nationwide basis are largely unknown in Germany. Methodology All German hospitals treating patients with ovarian cancer were asked to document all patients with first diagnosis in the third quarter in 2012 and 2016. Details of tumor, treatment and outcome were documented. This analysis is focusing on patients with AOC FIGO III/IV and the endpoint PFS (progression free survival) (OS data immature for 2016 cohort). As response rates were not documented, we defined a subcohort of patients without progressive disease within 3 months after end of chemotherapy as potential candidates for an additional maintenance therapy.

Result(s)* In total, 1010 patients with high grade AOC with a median age of 65 years were documented. 774/1010 (76.6\%) were diagnosed with FIGO III disease and 947/1010 (93.8\%) had high-grade serous histologic subtype. 915/1010 (90.6\%) had primary cytoreductive surgery. Complete resection was achieved in 434/1010 (43.0\%) at primary surgery and in 54/ $1010(5.3 \%)$ at interval debulking surgery. Carboplatin/paclitaxel/bevacizumab (TCB) was the primary systemic therapy in $627 / 1010(62.1 \%)$ and carboplatin/paclitaxel (TC) in 292/1010 (28.9\%). Median PFS in patients with primary surgery and complete resection was 29.7 months (95\% CI 27.4-34.2) compared to 17.2 months $(95 \%$ CI 15.7-18.9) in all other patients $(\mathrm{p}<0.001$ : HR 0.49 , 95\% CI 0.42-0.57). Median PFS in patients with TCB was 23.3 months (95\% CI 22.0-25.3) and 18.5 months (95\% CI 15.7-21.9) in patients treated with TC $(p=0,083$ : HR $0.87,95 \%$ CI 0.74-1.02). In total, 899/ 1010 (89\%) did not progress within 3 months.

Conclusion* Most patients in Germany with high grade AOC are treated with primary surgery followed by carboplatin/paclitaxel/bevacizumab. Depending on biomarker status most patients are potential candidates for a PARP-inhibitor maintenance therapy.

\section{COULD CHANGE IN NEOADJUVANT CHEMOTHERAPY REGIMEN AFTER 3 CYCLES LEAD TO BETTER OUTCOMES IN INOPERABLE OVARIAN CANCER?}

'S Adeleke*, 'N Brown, 'A Choy, 'Z Ahmed, 'A Tsirekidze, 'V Ramachandran, ${ }^{1} S$ Ramanaidu, 'A Haslam, 'J Galante, ${ }^{2} \mathrm{~S}$ Shi, 'S George, ${ }^{1} \mathrm{~A}$ Davis, ${ }^{1} \mathrm{C}$ Mikropoulos, 1J Waters, 'J Summers, 'R Jyothirmayi. 'Maidstone Hospital, United Kingdom; ' $G u y$ 's Hospital, United Kingdom

\subsection{6/ijgc-2021-ESG0.343}

Introduction/Background* Multiple studies have assessed the timing of surgery in high grade serous ovarian cancer (HGSOC). There is some evidence that neoadjuvant chemotherapy (NACT) followed by interval debulking surgery is non-inferior to primary cytoreductive surgery. The ICON $8 b$ study results are awaited to evaluate the use of upfront bevacizumab in these patients. However, there is currently limited evidence supporting switching chemotherapy regimens after 3 cycles of NACT when disease remains inoperable. In this retrospective study, we evaluated survival outcomes following a change of chemotherapy regimen in previously inoperable patients.

Methodology Patients with HGSOC treated with NACT from 2015 to 2020 were identified from 4 NHS trusts across Kent, UK. Chemotherapy regimens, number of cycles, debulking status/residual disease were extracted from electronic medical records. Kaplan-Meier analyses and Logrank tests were used to assess survival outcomes.

Result(s)* 149 patients were identified. 59\% (88/149) were deemed operable after 3 cycles of NACT (carboplatin + paclitaxel 3 weekly), with $39 \%(34 / 88)$ of these achieving optimal debulking. $41 \%(61 / 149)$ were inoperable after three cycles of NACT, with $79 \%(48 / 61)$ of these remaining inoperable after 